[3] T. Delbrück "Silicon Retina with CorrelationBased, Velocity-Tuned Pixels", IEEE Trans. Neural Networks, vol 4, pp. 529-541, 1993.

[4] R. Etienne-Cummings, S. Fernando, N. Takahashi, V. Shtonov, J. Van der Spiegel and P. Mueller, "A New Temporal Domain Optical Flow Measurement Technique for Focal Plane VLSI Implementation," in Proc. Comp. Arch. Machine Perception 1993, pp. 241-250, 1993

[5] P. Hasler, C. Diorio, B. A. Minch, C. Mead, "Single Transistor Learning Synapses" Advances in Neural Information Processing Systems 7, MIT Press, pp. 817-824, 1995 .

[6] T. Horiuchi, "An Auditory Localization and Coordinate Transform Chip" Advances in Neural Information Processing Systems 7, MIT Press, pp. 787$794,1995$.

[7] T. Horiuchi, B. Bishofberger, and C. Koch, "An Analog VLSI Saccadic System" Advances in Neural Information Processing Systems 6, Morgan Kaufmann, pp. 582-589, 1994.

[8] T. Horiuchi, J. Lazzaro, A. Moore and C. Koch, "A delay line based motion detection chip" in $A d$ vances in Neural Information Processing Systems 3, Morgan Kaufman, pp. 406-412, 1991.

[9] R. Jurgens, W. Becker, and H. H. Kornhuber, "Natural and Drug-Induced Variations of Velocity and Duration of Human Saccadic Eye Movements: Evidence for a Control of the Neural Pulse Generator by Local Feedback" Biol. Cybern. 39, pp. 87-96, 1981 .

[10] J. Kramer, "Compact Integrated Motion Sensor with Three-Pixel Interaction", submitted for publication to IEEE Trans. Pattern Anal. Machine Intell., May 1995.

[11] J. Kramer, R. Sarpeshkar and C. Koch, "PulseBased Analog VLSI Velocity Sensors," submitted for publication to IEEE Trans. Circuits and Systems II, April 1995.

[12] C. Mead, "Axons" in Analog VLSI and Neural Systems, pp. 193-204, Addison-Wesley, 1989.

[13] A. Moore and C. Koch, "A Multiplication Based Analog Motion Detection Chip" SPIE Vol. 1473 Visual Information Procesing: From Neurons to Chips, pp. 66-75, 1991.
[14] A. McKenzie and S. G. Lisberger, "Properties of Signals that Determine the Amplitude and Direction of Saccadic Eye Movements in Monkeys" $J$. Neurophysiol. Vol 56, No. 1, pp. 196-207, 1986.

[15] M. J. Nichols and D. L. Sparks, "Non-stationary Properties of the Saccadic System - New Constraints on Models of Saccadic Control", J. Neurophysiol. Vol 73 No. 1, pp. 431-435, 1995.

[16] L. M. Optican and F. A. Miles, "Visually Induced Adaptive Changes in Primate Saccadic Oculomotor Control Signals" J. Neurophysiol. Vol 54, No. 4, pp. 940-958, 1985 .

[17] L. M. Optican and D. A. Robinson, "CerebellarDependent Adaptive Control of the Primate Saccadic System" J. Neurophysiol. Vol 44, pp. 10581076. 1980 .

[18] D. A. Robinson, "Oculomotor Control Signals", in Basic Mechanisms of Ocular Motility and Their Clinical Implications, Pergamon Press, pp. 337-374, 1975 .

[19] R. Sarpeshkar, W. Bair and C. Koch "Visual Motion Computation in Analog VLSI Using Pulses", in Advances in Neural Information Processing Systems 5, Morgan Kaufman, pp. 781-788, 1993.

[20] R. Sarpeshkar, J. Kramer, G. Indiveri and C. Koch, "Analog VLSI Architectures for Motion Processing: From Fundamental Limits to System Applications", Submitted to: Proceedings of the IEEE

[21] J. Tanner and C. Mead, "An Integrated Analog Optical Motion Sensor," in VLSI Signal Processing, II, IEEE Press, 1986, pp. 59-76. pp. 20-30, 1982. 


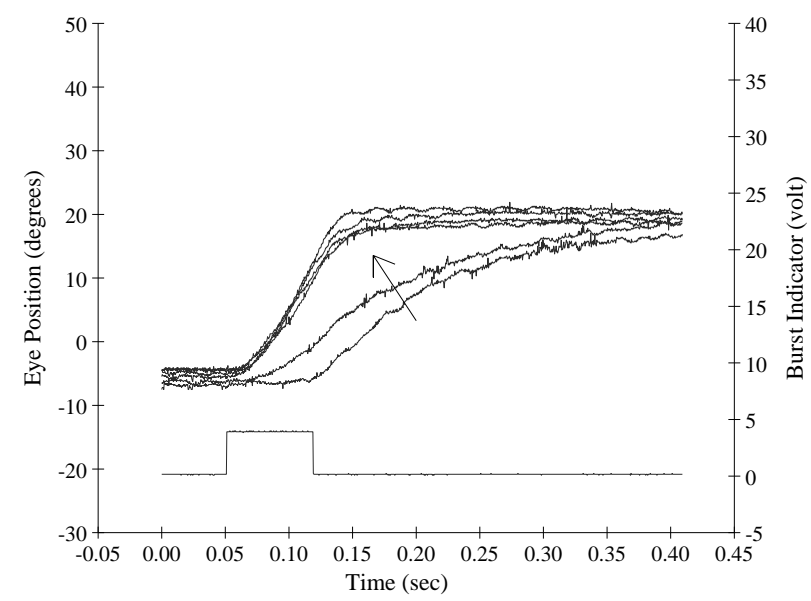

Figure 10. Saccade trajectories showing the reduction of an onward post-saccadic drift by increasing the burst gain via a tunneling process. See text for description. The lower trace indicates the period of the burst unit activity.

\section{6: Summary and Conclusions}

Using the one-dimensional saccadic eye movement system previously developed in our laboratory, we demonstrate the use of two new analog VLSI circuits to create an adaptive sensorimotor system. A new burst generator circuit has been presented which follows the current neurophysiological literature more closely and which incorporates floating-gate technology to provide non-volatile, local synaptic learning. A new directionselective motion detection circuit has been presented which has been used to guide the learning process. While we have thus far only addressed the reduction of post-saccadic drift, we anticipate the implementation of a system for the adaptation of saccadic accuracy.

The investigation of neural information processing architectures in analog VLSI can provide insight into the issues that biological nervous systems face. Ana$\log$ VLSI architectures share many of the advantageous properties with neural systems such as speed, space efficiency, and lower power consumption. In addition, analog VLSI must face similar constraints such as realworld noise, component variability or failure, and interconnection limitations. With the development of reliable floating-gate circuits, the powerful ability of neural systems to modify and store parameters locally can finally be realized in analog VLSI.

Beyond our effort to understand neural systems by building large-scale, physically-embodied biological

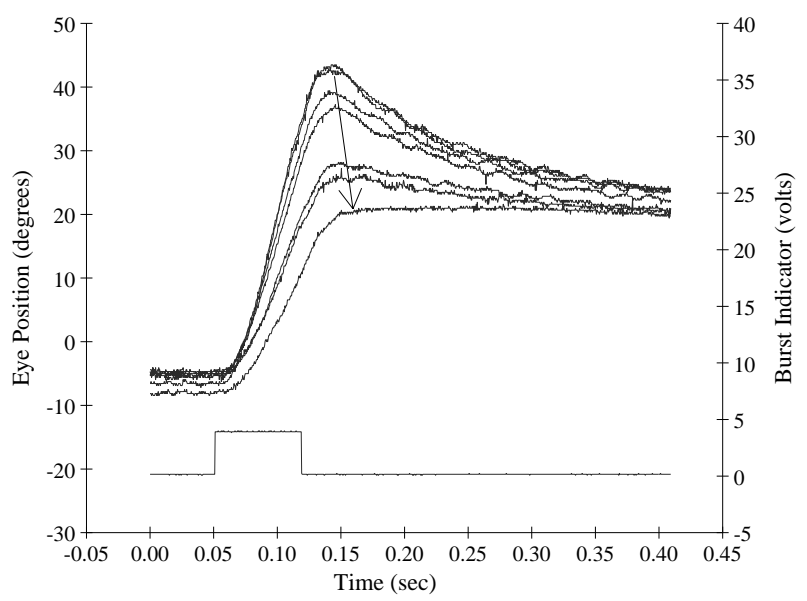

Figure 11. Saccade trajectories showing the reduction of a backward post-saccadic drift by decreasing the burst gain via a hot-electron injection process. See text for description. The lower trace indicates the period of the burst unit activity.

models, adaptive analog VLSI sensorimotor systems can be applied to many commercial and industrial applications involving self-calibrating actuation systems. In particular, we believe that for real-world tasks such as mobile robotics or remote sensing, these circuits will be invaluable for systems trying to keep up with the ever-changing world.

\section{7: Acknowledgements}

The authors would like to thank Paul Hasler for assistance in the design of the floating-gate circuits. This work is supported by an Office of Naval Research AASERT grant and by the NSF Center for Neuromorphic Systems Engineering at Caltech.

\section{References}

[1] A. G. Andreou, K. Strohbehn and R. E. Jenkins, "Silicon retina for motion computation" in Proc. 1991 IEEE Int. Symp. Circuits and Systems, Singapore, pp. 1373-1376, June 1991.

[2] R. G. Benson and T. Delbrück, "Direction Selective Silicon Retina that uses Null Inhibition", in $A d$ vances in Neural Information Processing Systems 4, Morgan Kaufman, pp. 756-763, 1992. 


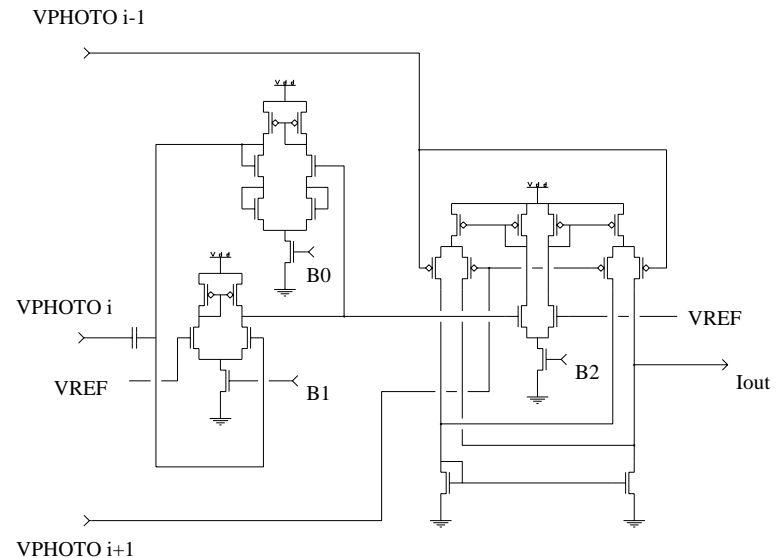

Figure 8. Circuit for computing the direction of visual motion. lout $=($ temporal derivative $)$ - (spatial derivative). The two transconductance amplifiers on the left are configured as a current-sense amplifier, providing the temporal derivative w.r.t. a reference voltage. This differential signal is fed into the Gilbert multiplier on the right. The other differential input comes from the two neighboring photoreceptors to provide the spatial derivative. Biases $B 1$ and $B 2$ are strong while B0 is set weakly and determines the sensitivity of the circuit.

\section{5: Training Results}

To demonstrate the learning, the direction-selective motion detector chip was mounted on the onedimensional eye and motion information was read from the chip $100 \mathrm{~ms}$. after the end of the burst activity. The burst activity period (lower trace in Figures 10 and 11) is detected by a signal representing the suppression of the pause circuitry on the burst generator chip. A standard leftward saccade amplitude of about 23 degress was programmed into the burst generator input and a saccade was triggered. The motion sensor was facing a stripe stimulus which would elicit a motion signal during and after the saccade burst.

Direction-of-motion information was integrated from across the array and a simple bang-bang type of learning algorithm was used to determine which direction to change the gain. $100 \mathrm{~ms}$. after each trial saccade, integration of the motion detector information yielded a scalar value which was compared against two threshold values. If the integrated value was greater than the rightward motion threshold, indicating over-

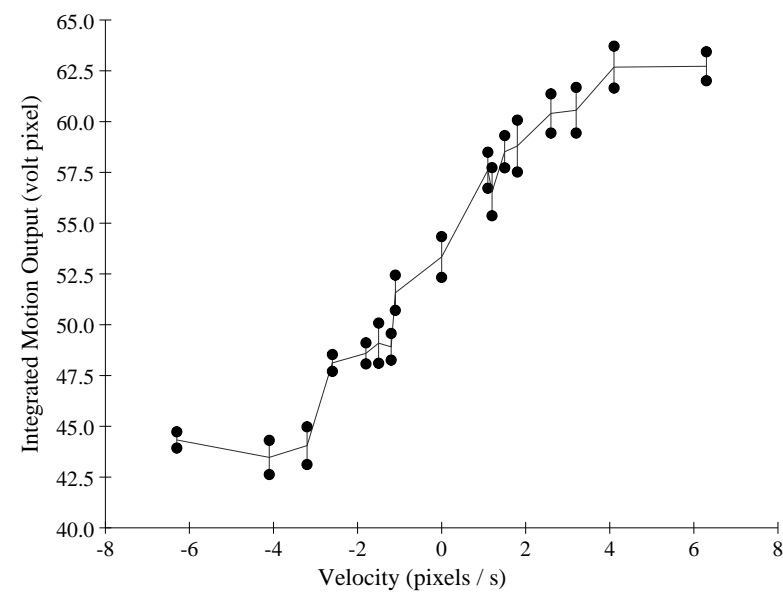

Figure 9. Integrated motion output signal vs. stimulus velocity. Note that the specific value of the integrated output is also dependent on the contrast and spatial frequency of the stimulus. The stimulus used was a high-contrast grating which fit 3 bars onto the 20 pixels of the array. The lowest measured stimulus speed was at 1.1 pixels $/ \mathrm{s}$. The vertical bars represent one standard deviation. shoot, a unit hot-electron injection pulse was issued which would reduce the floating-gate voltage and thus reduce the burst gain. If the integrated value was less than the leftward motion threshold, indicating undershoot, a unit tunneling pulse was issued which would increase the floating-gate voltage and thus increase the pulse gain. At present, the activation of both tunneling and injection pulses is performed manually, however, the criterion for the direction of the adaptation pulse is strictly based on the scalar value reported by the motion detection chip and could be automated.

Figure 10 shows an experiment where the pulse gain was initialized to a value near zero. Within 8 trials (not all traces are shown), the pulse gain was raised sufficiently to eliminate the post-saccadic drift. Figure 11 shows a similar experiment where the pulse gain was initialized to large value. In this case, 41 trials (not all traces are shown) were required before the gain was reduced sufficiently to eliminate the post-saccadic drift. The difference in learning rates is not important and can easily be balanced. 


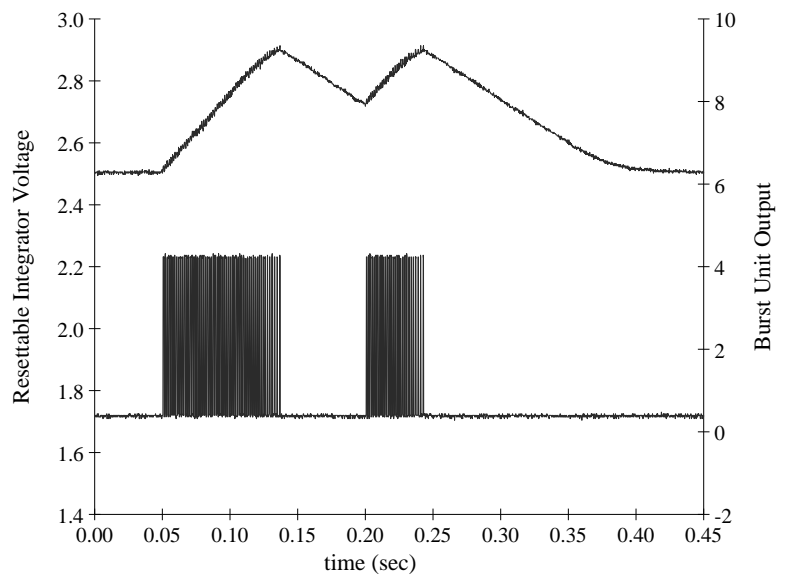

Figure 6. Due to the reset latency of the burst integrator (upper trace), burst commands issued before the integrator has fully reset result in reduced amplitude saccades which vary systematically with command latency. See experiments by Nichols and Sparks 1995 [15]

was 10 seconds. Adaptation was performed between saccades, following evaluation of the motion circuit.

\section{4: A Direction-Selective Motion Detec- tor}

In order to reduce the post-saccadic drift, full-field visual motion is required for the adaptation error signal. Both gradient models and correlation models for computing short-range visual motion have been explored in analog VLSI technology, and many designs have focused on computing the velocity at each pixel $[21],[8],[19],[4],[11],[10]$. In certain applications, however, the direction of the motion alone can be used, often resulting in a simpler circuit [1],[2],[3],[13]. (For a review of analog VLSI motion detectors see Sarpeshkar et al. [20].)

In particular, tasks where the visual motion is used to determine an error signal for some adaptive sensorimotor control system, the direction of motion is often sufficient for guiding a learning process. The problem posed here of reducing post-saccadic drift falls into this category. The particular task of correcting postsaccadic drift requires a visual motion signal that is sensitive to very low velocities and accurately reports the sign. The lowest detectable velocity will thus determine the lower limit of correctable drift.

If direction and sensitivity are the most impor-

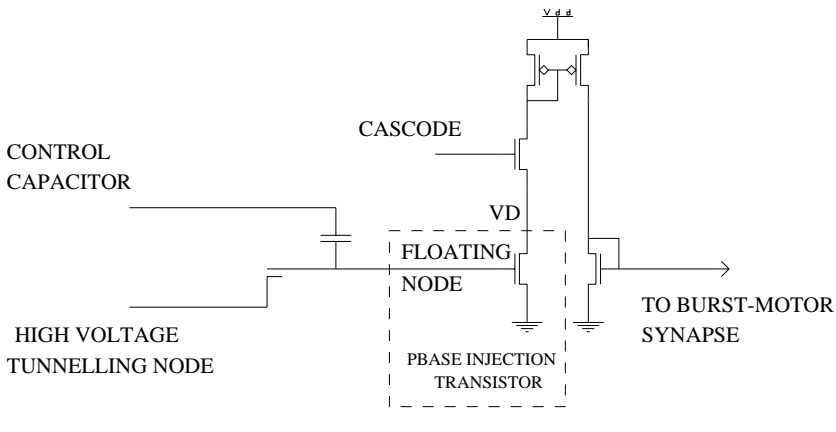

Figure 7. The floating-gate device used for storage of an analog current reference. The floating node potential is raised by tunneling electrons off of the node with high voltage and lowered by injecting electrons onto the node via the pbase injection transistor. The cascode transistor controls the drain voltage (VD) which is used to modulate the hot-electron injection process. The current flowing through the mirror is used as the output.

tant features of the motion detector required, then the product of the temporal derivative $\left(I_{t}\right)$ and spatial derivative $\left(I_{x}\right)$ gives an appropriate signal. Similar in aim to the analog VLSI-based circuit implemented by Moore and Koch [13], the result is an output which is velocity $I_{x}^{2}$. Two benefits of this circuit are: 1 ) the lower limit of velocity is limited by the sensitivity and noise of the temporal derivative circuit, and 2) the output goes to zero as the image's spatial derivative goes to zero, thus avoiding the divide-by-zero problem in the gradient-model.

Figure 8 shows the circuit used to compute $I_{t} \cdot I_{x}$. See the caption for a description. A linear array of 20 elements has been fabricated on a TinyChip in a $2 u m$ n-well CMOS process through the MOSIS Service. The motion circuit width on the chip is $63 \mu \mathrm{m}$.

Since the circuit reports velocity $\cdot I_{x}^{2}$ the output depends on the stimulus used. Although the circuit computes the direction of motion at each pixel, it is possible to integrate information from across the entire array to obtain a better estimate of a full-field motion stimulus such as post-saccadic drift. In order to quantify the performance of the circuit, the motion outputs from across the array were summed and compared for different speeds. Figure 9 shows this data for a drifting sinewave stimulus which had 3 full cycles visible on the array. 


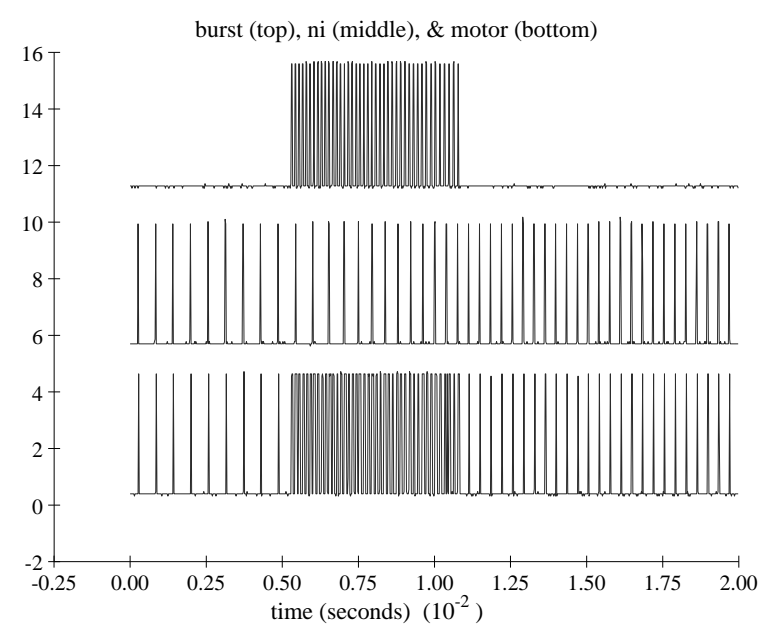

Figure 4. The burst, neural integrator, and motor units' output during a small saccade. The initial eye position is off-center with the neural integrator providing the tonic holding activity.

the burst generator chip, the pause circuitry is implemented by a bistable circuit which is turned off by the trigger signal and turned on by the comparator once the burst is over. The reset follower circuit (Fig 3, upper left) is also controlled by this circuit, discharging the integrator to the reset value when the burst is completed.

A pulse-rate representation for the various signals was chosen primarily to simplify the synaptic multiplication circuit down to two transistors, one for switching and one for setting the synaptic current. Although ideal for driving motors directly, the motor output pulses in this case are not used in this way, due to the need to combine these control signals with the signals used to generate the dynamics of the eye. An example set of saccade signals are shown in Figure 4. Eye movement traces for three saccades with identical inputs and tonic components but different burst gains are shown in Figure 5.

Recent experiments by Nichols and Sparks [15] have demonstrated a recovery period associated with the burst generator consistent with the resettable integrator model. Figure 6 mimics the experimental result with two closely-spaced identical saccade commands. The upper trace shows the integrator voltage as it charges up during the burst and then discharges between saccades.

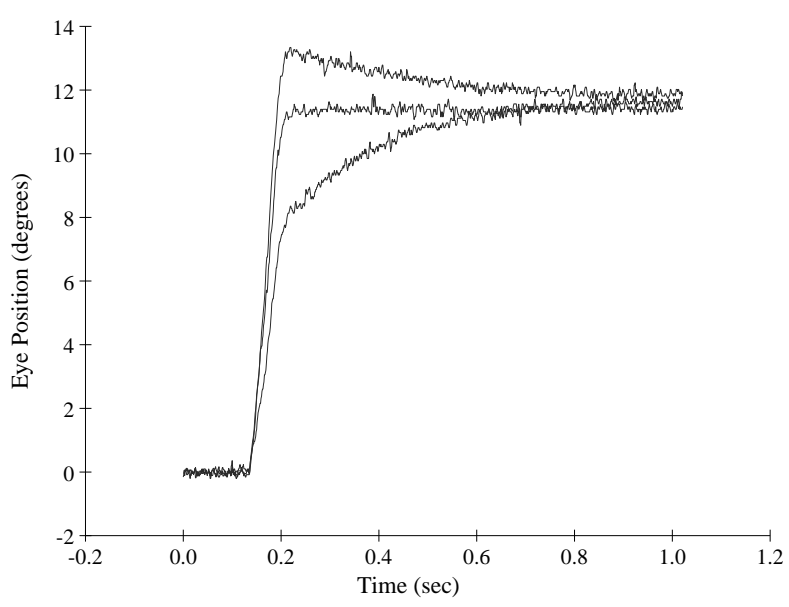

Figure 5. Eye movement traces for $\mathbf{3}$ small saccades with the same input command and tonic component gain but with different burst component gains. The upper trace shows an example of an overshoot condition caused by excessive gain and the lower trace shows an example of an undershoot condition caused by insufficient gain. Note that the overshoot case generates a backward post-saccadic drift and the undershoot case generates an onward postsaccadic drift. The middle trace is an example of a properly set gain. This step shape is what the adaptation system is trying to achieve.

\section{3: Non-volatile Analog Voltage Storage}

A floating-gate device [5] is used to store the gain parameter for the connection between the burst unit and the motor unit. The circuit used in this system is shown in figure 7 . This device utilizes high-voltage tunneling in order to remove electrons from the floating node and hot-electron injection to put electrons onto the floating node. The tunneling occurs through a high-quality gate oxide between a well-region and the Polyl layer floating node. The hot-electron injection is performed using a pbase substrate transistor. For details please see Hasler et al. [5]

For the data presented in this paper, the tunneling was operated by driving the high-voltage node from 25 volts up to 33 volts. Injection was controlled by moving the cascode transistor gate from 1.50 volts up to 4.28 volts. The cascode technique allows control over the drain voltage (VD) of the injection transistor which modulates the rate of injection. The standard pulse length of both the injection and tunneling processes 


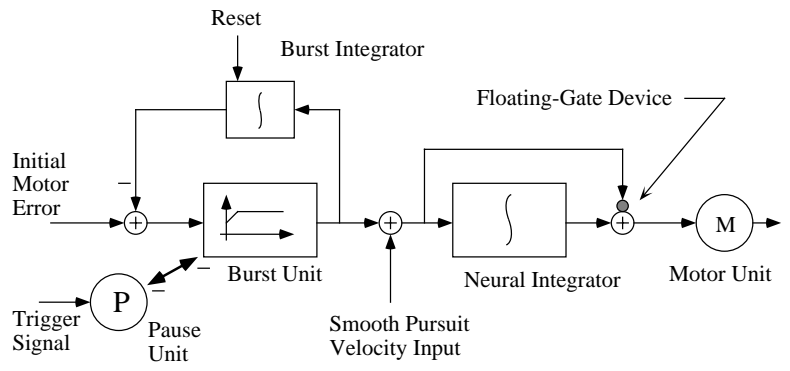

Figure 2. System block diagram of the burst generator, neural integrator, motor unit, and pause units. This system generates the motor signals used to drive the motors in the model oculomotor plant. Initially, the neural integrator is providing a tonic signal to the motor unit to hold the eye in its starting position. The initial motor error input to the system (far left) is applied and a trigger signal disinhibits the burst unit via the pause unit. The burst integrator activity (starting from zero) rises and is compared against the input activity to drive the burst unit. When the difference goes to zero, the pause unit shuts down the burst. Downstream, the burst unit provides the burst component and is also integrated via the neural integrator to provide the tonic component. These two signals are combined at the input to the motor unit. The parameter being trained in this system is burst gain (floating-gate).

The control of saccadic eye movements is further complicated by the long latency at which proprioceptive or visual information becomes available. For a small amplitude saccade, the movement is over before sensory information is available that the movement has even started. The saccadic burst generator is therefore believed to operate in an open-loop mode of control [18], issuing a motor program whose parameters are adjusted between saccades.

While many detailed biological models of this system have been simulated, and many hardware systems exhibiting quick eye movements have been built, we have constructed a saccadic eye movement system implementing various biological models in analog VLSI circuitry which drive an oculomotor plant exhibiting the appropriate dynamics [7]. In previous work we have demonstrated the use of visual and auditory targets [6] to trigger target-centering saccades.

In this paper we demonstrate a system which re- duces the post-saccadic drift based on detected visual motion. First, we introduce a new burst generator circuit and its performance driving the eye. Second, we discuss a floating-gate device and how it is used for on-chip storage. Third, we introduce a new motion detection circuit for detecting post-saccadic drift, and finally, we combine them into a single system which is capable of improving its performance through repeated trials based on a visually-detected error signal.

\section{2: The Resettable-Integrator Burst Gen- erator Chip}

The burst generator model used in this system (See Figure 2) is a double integrator model based on the work by Jürgens et al. [9] and MacKenzie and Lisberger [14] which uses initial motor error as input to the system and requires a reset period following the saccade.

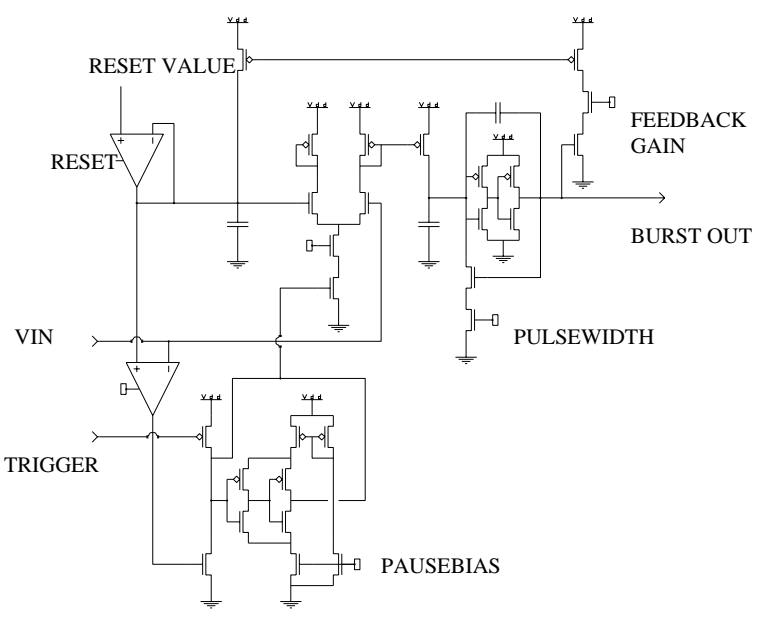

Figure 3. The burst generator circuit for one direction. The circuit consists of a differential pair which compares the input motor error (VIN) with the local integrator value. If the input motor error is larger than the input, then current flows into a neuron circuit [12] which fires off pulses, charging up the integrator via the feedback pathway until the integrator voltage matches VIN. The comparator output then pulls the bistable pause circuit low, shutting down the burst and activating the reset follower.

The basic operation of the model is described in Figure 2. The circuit which implements the burst generator model for one direction is shown in Figure 3. On 


\title{
Analog VLSI Circuits for Visual Motion-Based Adaptation of Post-Saccadic Drift
}

\author{
Timothy K. Horiuchi \& Christof Koch \\ Computation and Neural Systems Program \\ California Institute of Technology \\ Pasadena, CA, 91125 \\ E-mail: timmer@klab.caltech.edu
}

\begin{abstract}
Using the analog VLSI-based saccadic eye movement system previously developed in our laboratory [7], we are investigating the use of biologically realistic error signals to calibrate the system in a manner similar to the primate oculomotor system. In this paper we introduce two new circuit components which are used to perform this task, a resettable-integrator model of the burst generator with a floating-gate structure to provide on-chip storage of analog parameters and a directionally-selective motion detector for detecting post-saccadic drift.
\end{abstract}

\section{1: Introduction}

The most common eye movement in primates are the quick reorienting movements known as saccades. Our eyes often reach speeds up to $750 \mathrm{deg} / \mathrm{s}$ during a

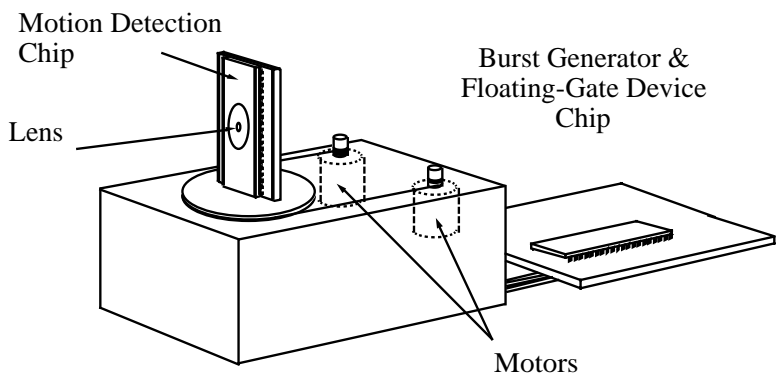

Figure 1. Drawing of the motion detector chip mounted on the one-dimensional eye with the burst generator chip mounted on a different board. saccade which severely impairs our visual acuity. It is therefore important to minimize the time during which the eye is moving. While typical human saccades last between $40 \mathrm{~ms}$. and $150 \mathrm{~ms}$, changes or damage to either the oculomotor plant or the underlying neural circuitry can cause significant eye drift following a saccade, lengthening the period of poor acuity.

In the biological oculomotor plant, the eye is suspended in the eye socket both by suspensory tissues and the eye muscles which create an overdamped springmass system. In order to hold the eyes off-center it is therefore necessary to provide a sustained pulling force on the muscles to counter the elastic properties of the muscles which would passively return them to the center position. In addition, quick eye movements require a large, transient, driving force to overcome the long time-constant of the system due to the viscosity in both the muscles and the eye socket.

Since the sustained (or tonic) component of the command determines the final eye position, the ideal transient (or burst) component should bring the eye to exactly that position by the end of the burst. Mismatch of the burst and tonic components leads to either an onwards or backwards drift following an undershoot or overshoot of the final eye position. This motion is known as post-saccadic drift. Studies in both humans and monkeys show that these deficits can be compensated for by some type of learning process which have time constants on the order of 1.5 days. Ablation studies [17] have further shown that control of the burst and tonic gains is independent and that their control depends on different areas of the cerebellum. While two different afferent systems could potentially be used to report the presence of ocular drift (proprioceptive inputs and full-field retinal slip), retinal slip was shown to be sufficient to elicit these adaptive changes and compensate for leftward and rightward saccades.[16]. 